\title{
Necrotizing enterocolitis in a mouse model leads to widespread renal inflammation, acute kidney injury, and disruption of renal tight junction proteins
}

\author{
Parvesh M. Garg', Rodney Tatum², Srikanth Ravisankar' , Prem S. Shekhawat ${ }^{1,3}$ and Yan-Hua Chen ${ }^{2}$
}

BACKGROUND: Necrotizing enterocolitis (NEC) is a devastating condition affecting premature infants and leads to high mortality and chronic morbidity. Severe form of NEC is associated with acute renal failure, fluid imbalance, hyponatremia, and acidosis. We investigated the effect of NEC on tight junction (TJ) proteins in kidneys using a NEC mouse model to investigate the basis for the observed renal dysfunction.

METHODS: NEC was induced in C57BL/6 mice by formula feeding and subjecting them to periods of hypoxia and cold stress. NEC was confirmed by gross and histological examination. We studied various markers of inflammation in kidneys and investigated changes in expression of several TJ proteins and AQP2 using immunofluorecent staining and western blotting.

RESULTS: We found markedly increased expression of NFKB, TGF $\beta$, and ERK1/2 along with claudin-1, -2, -3, -4, -8, and AQP-2 in NEC kidneys. The membrane localization of claudin-2 was altered in the NEC kidneys and its immunostaining signal at TJ was disrupted.

CONCLUSION: NECled to a severe inflammatory response not only in the gut but also in the kidneys. NEC increased expression of several TJ proteins and caused disruption of claudin-2 in renal tubules. These observed changes can help explain some of the clinical findings observed in NEC.

$\mathbf{N}$ ecrotizing enterocolitis (NEC) is a commonly witnessed gastrointestinal emergency of the premature infants (birth weight $<1,500 \mathrm{~g}$ ), and is characterized by transmural coagulative necrosis, bacterial overgrowth, pneumatosis, and severe intestinal inflammatory response (1-3). NEC affects nearly $6-10 \%$ of low-birth-weight infants in any neonatal intensive care unit and leads to mortality in $15-30 \%$ of subjects and chronic morbidity in survivors $(4,5)$. NEC leads to poor neurodevelopmental outcomes among survivors and estimated cost of caring for these infants range between 500 million to 1 billion dollars each year (3). Although the pathogenesis of NEC is still not well understood, it is considered multifactorial with prematurity, enteral feeding, and abnormal bacterial colonization of the gut being the major risk factors.

Onset of NEC is intimately related to breach of the gut epithelial barrier with resultant change in intestinal permeability to foreign proteins, endotoxins, and translocation of luminal bacteria into the circulation (6-10). Intestinal permeability is tightly regulated by several tight junction (TJ) proteins, especially the claudin group of proteins, which are a family of 24 members (molecular weight: $20-27 \mathrm{kD}$ ) with four transmembrane domains $(9,11)$. Endotoxins acting via NF- $\kappa B$ pathway have been shown to alter TJs and protein expression in kidneys (12). Claudins show a tissue-specific distribution pattern and are expressed on epithelial linings of both the gastrointestinal tract and nephrons (13-17). Claudins can form either paracellular size- and charge-selective pores or paracellular ion barriers to control transport across epithelial linings (18-22). Thus claudins play an intimate role in maintenance of the epithelial barrier and protect infants from development of NEC.

Likewise claudins and aquaporins play a significant role in maintenance of normal renal function. Paracellular ion transport, which is a passive process, occurs through pathways formed by claudins but it is driven by transepithelial electrochemical gradient. The paracellular permeability and ionic conductance of TJs vary along the length of nephron, with a decrease in overall leakiness from the proximal tubules toward the collecting ducts (23), which could be explained due to the differential transport processes and differences in driving forces along the nephron segments. The proximal renal tubules which are more leaky segments of the nephron express the channel-forming claudins (e.g., claudin-2 and -10), while the distal nephron which has reduced paracellular permeability and solute transport typically expresses the sealing claudins (e.g., claudin-4, -8, and -14) (24-26).

Severe cases of NEC, especially those associated with high mortality, present clinically with a septic-shock like condition with acute renal failure, hyponatremia, and metabolic acidosis $(3,5)$. These metabolic changes cannot solely be explained by intestinal inflammation and necrosis with subsequent 
generalized edema but are likely mediated through changes in renal function. We hypothesized that kidneys are affected by this gut inflammatory process and renal TJ proteins are affected in NEC. We used a neonatal NEC mouse model to investigate the effect of NEC on kidney TJ proteins. Our observations provide the experimental basis to help explain the reason behind the acute renal failure, fluid imbalance, and hyponatremia, which is commonly seen in neonates with severe NEC.

\section{RESULTS}

\section{Histological Examinations of Intestines and Kidneys}

After 4 days of formula feeding and stress treatment, about $50 \%$ of mice developed NEC compared to none in the dam fed group. As shown in Figure 1a, the NEC pups were much smaller in size compared to the control pups and showed clinical signs of disease. The NEC intestines were swollen in appearance and developed hemorrhagic necrosis as indicated by the arrow in Figure $\mathbf{1 b}$ in comparison to control intestines. The NEC phenotype was confirmed by histopathology examination using standard criteria (27). Intestinal sections from control pups displayed intact villi and submucosa (Figure 2a) with minimal mononuclear cells in the villi. However, the intestinal sections from NEC pups showed disrupted villi and submucosa with intense mononuclear cell infiltration. Some villi showed complete sloughing of epithelial cells with breach of epithelium (Figure 2b, arrow).

a

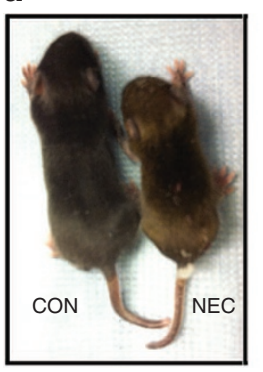

b
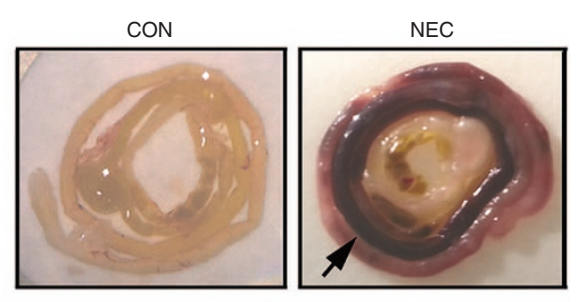

Figure 1. Generation of necrotizing enterocolitis (NEC) mouse model. (a) Four-day-old pups were either dam-fed (CON) or fed with Esbilac formula and underwent asphyxia and cold stress treatments twice a day for $4 \mathrm{~d}$ (NEC). The NEC pup is much smaller than the control pup. (b) The control and NEC intestines were rolled up as a Swiss roll. Compared to the control intestine, the NEC intestine shows the severe hemorrhage (arrow).
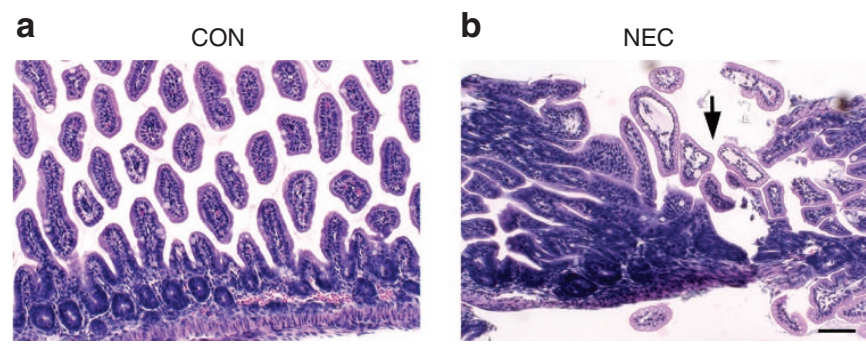

Figure 2. Histopathology of small intestine sections (H\&E staining) from control and necrotizing enterocolitis (NEC) pups. (a) Representative control sample shows the normal villous structure with intact crypt region. (b) The induction of NEC led to the disruption of villous structure (arrow). Scale bar: $100 \mu \mathrm{m}$ for (a) and (b).
Kidneys from pups whose intestines were showing NEC histological grading $>2$ were grossly normal in appearance but on histology showed interstitial edema and mononuclear interstitial infiltrates as compared to the kidneys from the control group (Figure 3b,d, arrows). The increased infiltrating immune cells in the NEC kidneys were also confirmed by the immunostaining of the $\mathrm{CD} 11 \mathrm{~b}$ antibody that recognizes the monocytes and macrophages (Figure 3e). There was no global or segmental sclerosis and no interstitial fibrosis in either group of renal sections, ruling out any pre-existing renal pathology.

\section{Altered Expression and Localization of TJ Proteins and Water Channel AQP2 in NEC Kidneys}

We found increased expression levels of claudin-1, -2, -3, -4, and -8 in NEC kidneys on western blot analysis compared to controls (Figure 4). Immunofluorescent staining method was used to examine the localization of several claudin proteins (Figure 5). We noted increased signals of claudin-1 and -2 in the NEC kidneys (Figure 5b,d). Claudin-2 is known to be located in the proximal tubules of kidneys and responsible a

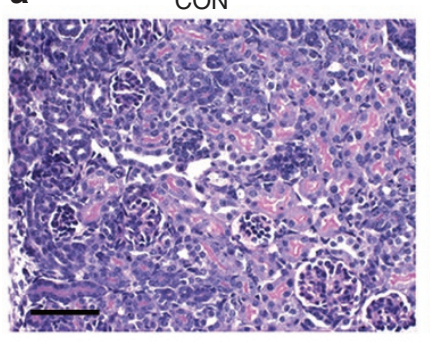

c

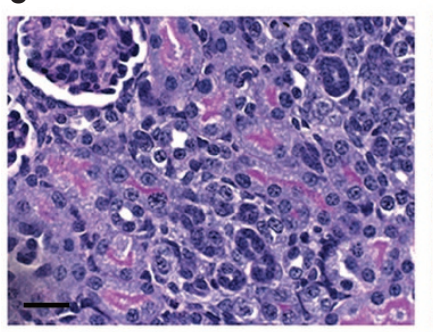

e

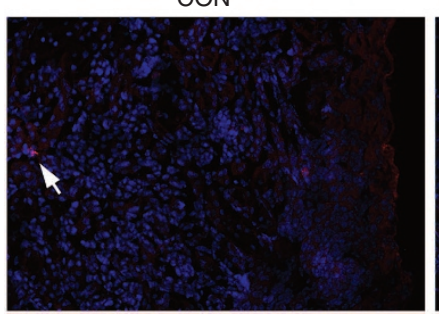

b

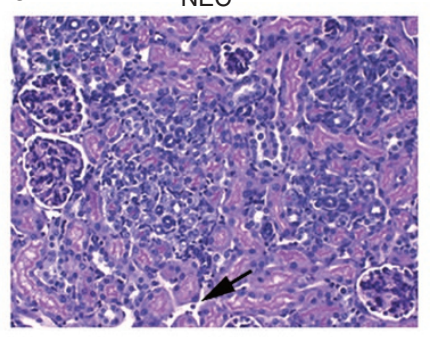

d

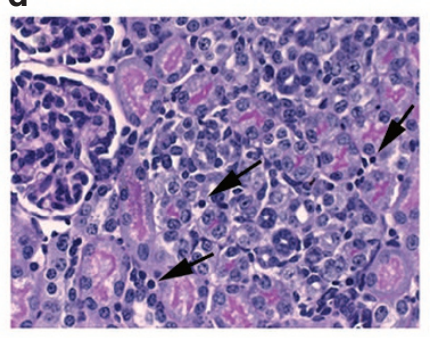

NEC

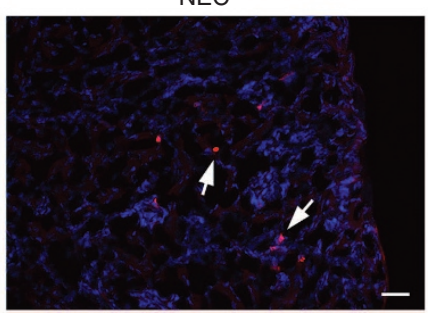

Figure 3. Histopathology of kidneys and the infiltration of immune cells. The control $(\mathbf{a}, \mathbf{c})$ and necrotizing enterocolitis (NEC) $(\mathbf{b}, \mathbf{d})$ kidney sections from cortex regions (H\&E staining) show normal glomerular and tubular architecture. However, NEC kidneys display the interstitial edema and mononuclear cell infiltration (arrows). (e) Frozen sections of control and NEC kidneys were stained with the CD11b antibody (red) recognizing monocytes and macrophages (arrows). The hoechst (blue) was used to stain nuclei. Scale bar: (a) and (b), $100 \mu \mathrm{m}$; (c) and (d), $20 \mu \mathrm{m}$; (e), $60 \mu \mathrm{m}$. 
for paracellular $\mathrm{Na}^{+}$reabsorption (20). Interestingly, claudin-2 subcellular localization was altered in the NEC kidneys. Its TJ staining pattern was disrupted compared to that of the control kidneys (Figure 5c,d, arrows), while claudin-1 and -7 showed the conventional TJ staining pattern for both control and NEC kidneys (Figure 5a,b,e,f, arrow). The NEC kidneys also showed increased expression of water channel AQP2 by western blot analysis (Figure 6a) as well as by immunofluorescence staining a

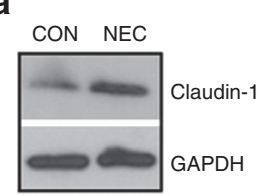

d

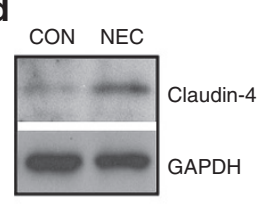

g b
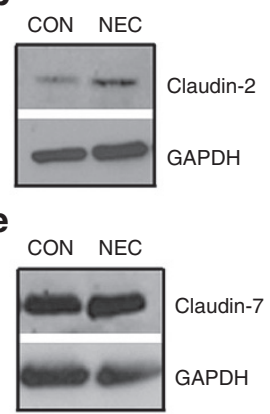

C
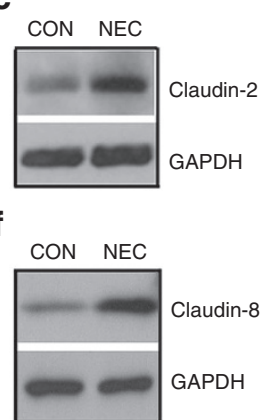

CON NEC

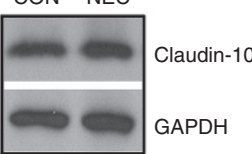

h

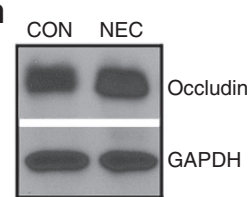

Figure 4. Expression levels of tight junction proteins in control and necrotizing enterocolitis kidneys. Kidney tissues were homogenized and lysed in the lysis buffer. A total of $30 \mu \mathrm{g}$ protein for each sample were loaded on the sodium dodecyl sulfate-polyacrylamide gel electrophoresis. Membranes were blotted against claudin-1 (a), -2 (b), -3 (c), -4 (d), -7 (e), $-8(\mathbf{f}),-10(\mathbf{g})$, and occludin (h) antibodies. GAPDH served as a loading control.
(Figure 6b) as compared to control kidney samples. The statistical analysis shows that both the number of the AQP2 signal and the intensity of the signal were significantly increased in the NEC kidneys (Figure 6c,d).
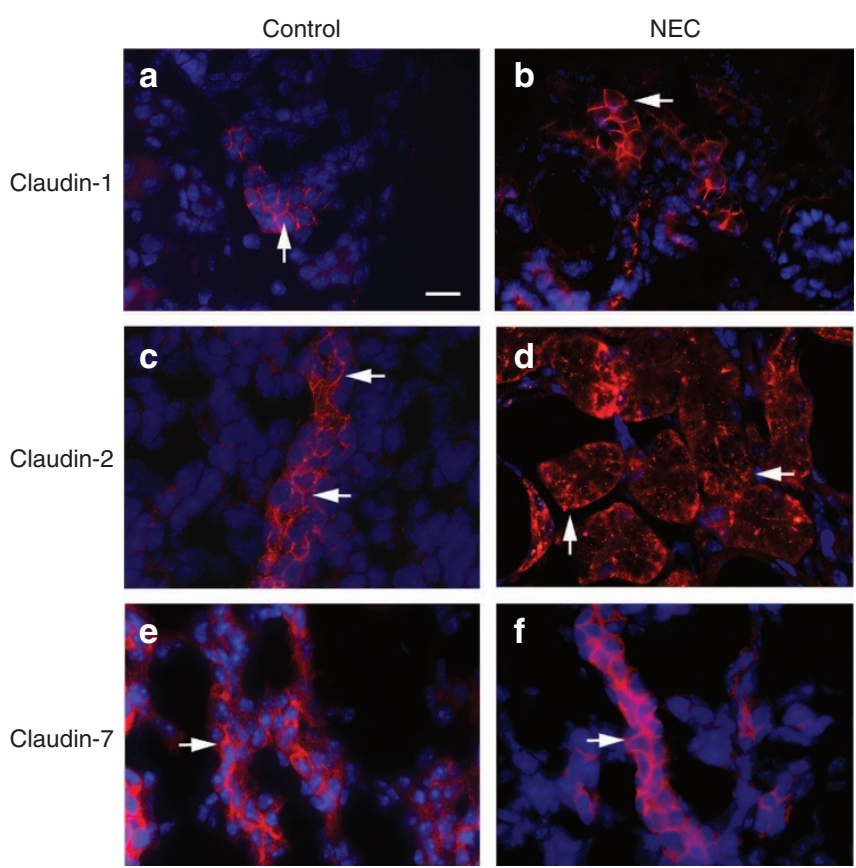

Figure 5. Localization of claudin-1, -2 , and -7 in control and necrotizing enterocolitis (NEC) kidneys. Control and NEC kidneys were removed from the body and frozen in liquid nitrogen. Frozen sections ( $5-\mu \mathrm{m}$ thickness) were immunostained with anti-claudin-1 (a,b), $-2(\mathbf{c}, \mathbf{d})$, and -7 (e,f) antibodies. Arrows in $\mathbf{a}, \mathbf{b}, \mathbf{c}, \mathbf{e}$, and $\mathbf{f}$ indicate the conventional TJ staining and arrows in $\mathbf{d}$ point to the altered staining pattern. Scale bar: $20 \mu \mathrm{m}$. a

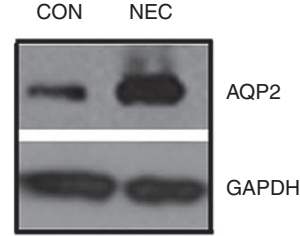

b
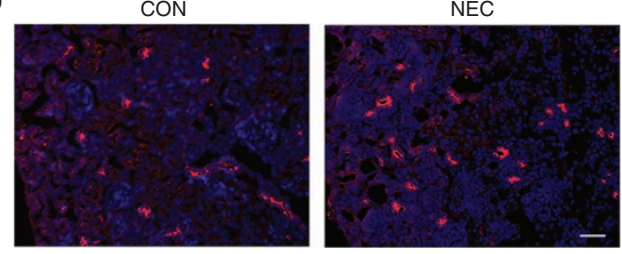

d

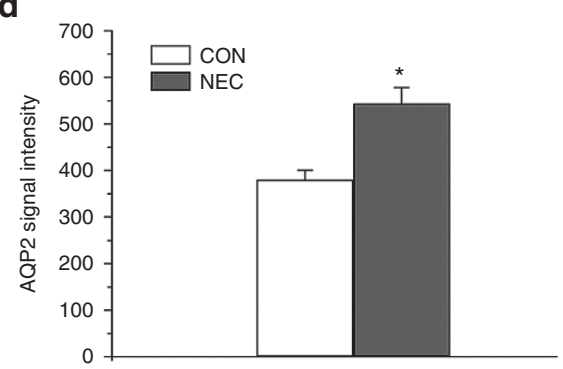

Figure 6. Expression of AQP2 in control and necrotizing enterocolitis (NEC) kidneys. (a) Kidneys from control (CON) and NEC pups were homogenized and lysed in the RIPA buffer. A total of $30 \mu \mathrm{g}$ protein for each sample were loaded on the SDS-polyacrylamide gel. Membranes were blotted against AQP2 antibody. GAPDH served as a loading control. (b) Frozen sections ( $5-\mu m$ thickness) of control (CON) and NEC kidneys were immunostained with anti-AQP2 antibody and detected by Cy3-conjugated anti-rabbit secondary antibody. The hoechst (blue) was used to stain nuclei. Scale bar: $50 \mu \mathrm{m}$. (c) The number of AQP2 signal on each image field was counted by AxioVision imaging software. Twelve images of control and NEC kidney samples were randomly selected for statistical analysis. *Value is significantly different from the control $(P<0.05)$. (d) The AQP2 signal intensity was measured by AxioVision imaging software. Sixty individual AQP2 signals from control and NEC kidneys were randomly selected for statistical analysis. ${ }^{*}$ Value is significantly different from the control $(P<0.05)$. 


\section{Elevation of Markers of Inflammation in NEC Kidneys}

Histological examination of NEC kidneys revealed a markedly increased number of immune cells compared to controls (Figure 3). On western blot analysis, we found elevated expression levels of TGF $\beta$ and NFKB p65 as well as ERK1/2 and p38 in NEC kidneys compared to control kidneys (Figure 7a). We also observed an increased number of apoptotic cells in NEC kidneys on terminal deoxynucleotidyl transferase dUTP nick end labeling (TUNEL) staining, indicating that NEC leads to acute kidney injury (Figure $7 \mathbf{b}$ ). The apoptotic cells were located on the tubular epithelial cells labeled with occludin, a TJ membrane protein marker (Figure 7c, arrows).

\section{DISCUSSION}

Our data provides evidence of renal inflammation with changes in expression of several TJ proteins and water channel secondary to gut injury in an experimental mouse model of NEC. To our knowledge this is the first report to demonstrate this effect of renal inflammation and changes in TJ proteins after onset of NEC, though there are several reports of disruption of TJ proteins in the GI tract (7), in cisplatin-induced nephrotoxicity (28) and in experimental type 1 diabetic nephropathy (29). Endotoxemia (12) and cisplatin treatment (28) have been shown to alter TJ protein expression in kidneys, but we did not find any reference to similar findings in NEC cases. NEC with associated gut inflammation leads to extravasation of intestinal contents including luminal pathogens across the epithelial barrier leading to systemic inflammation and septicemia. Whether this inflammatory process can extend to the kidneys in cases of NEC has not been recognized in the past, therefore these findings open the possibility of newer treatment approaches to this life-threatening complication of NEC. Severe cases of NEC are frequently associated with acute renal failure, hyponatremia, and acidosis which may last for several days to weeks and are associated with much higher mortality in affected neonates. Clinicians assumed that renal failure in NEC is related to a "shock like condition" with leaky capillaries and generalized edema resulting in decreased perfusion pressure of nephrons, thus leading to acute renal failure. The common approach in this situation is to provide fluid boluses followed by use of highly potent diuretics, even though there may also be acute kidney injury in these cases. Kidneys in severe cases of NEC have an altered function and lose their capacity to excrete waste products and water appropriately. Our histopathology studies using $\mathrm{H} \& \mathrm{E}$ staining revealed interstitial infiltration of glomeruli and tubules by abnormally higher numbers of mononuclear cells in NEC kidneys compared to control kidneys. In addition, we found increased expression of several inflammatory marker proteins, including NFKB p65 and TGF $\beta$ along with a marked increase in ERK1/2 and p38 signals on western blot analysis. Since ERK and TGF $\beta$ are considered protective molecules for the renal tissue, their upregulation may also be a reaction to the tissue injury seen in our mouse model of NEC. We found evidence of increased apoptosis on TUNEL staining in NEC kidneys. Thus NEC may lead to acute kidney injury in severe cases. a
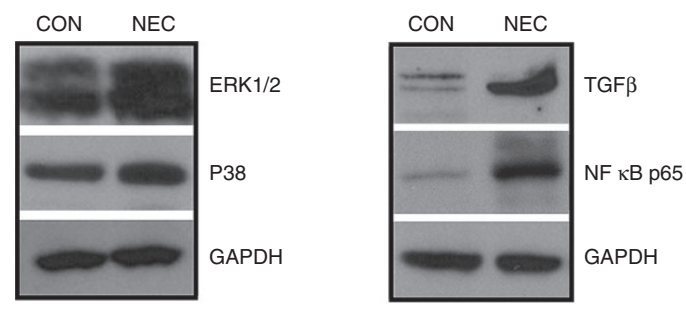

b

Tunnel
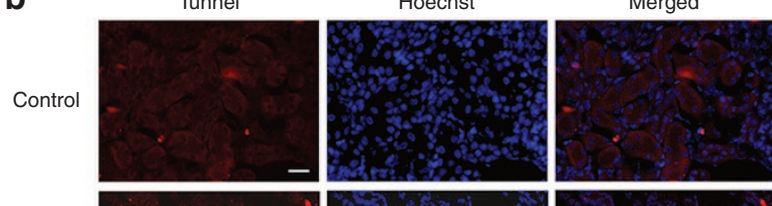

NEC
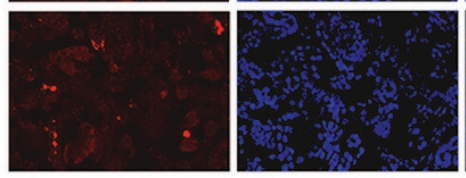

C
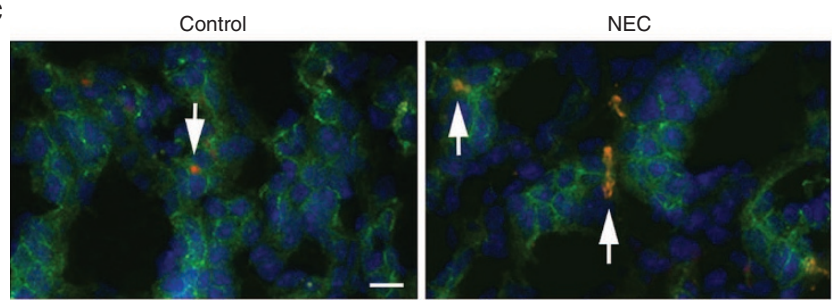

Figure 7. Increased levels of inflammatory marker proteins and apoptotic cells in necrotizing enterocolitis (NEC) kidneys. (a) Control and NEC kidney lysates were loaded on the SDS-polyacrylamide gel. Membranes were blotted against ERK1/2, p38, TGF $\beta$, and NFKB p65. GAPDH served as a loading control. (b) Control and NEC kidneys were fixed with $100 \%$ acetone and incubated with 10\% BSA in phosphate-buffered saline for $30 \mathrm{~min}$ at $37^{\circ} \mathrm{C}$ before applying terminal deoxynucleotidyl transferase dUTP nick end labeling (TUNEL) reaction mixture (Roche Diagnostics, Indianapolis, IN) to the tissues for $1 \mathrm{~h}$ at $37^{\circ} \mathrm{C}$. The red signal indicates the apoptotic cells. The blue signal is the nuclear staining. Scale bar: $50 \mu \mathrm{m}$. (c) Frozen sections from control and NEC kidneys were double stained with occludin (green) and TUNEL (red). Arrows indicate the apoptotic cells localized in occludin-positive tubular epithelial cells. The blue signal is the nuclear staining. Scale bar: $20 \mu \mathrm{m}$.

We observed increased expression levels of several TJ proteins, namely claudin- $1,-2,-3,-4$, and -8 in our experimentally induced NEC mouse pup's kidneys by western blot analysis. Claudin proteins selectively form paracellular channels or barriers. It is known that claudin-1 is largely expressed at the TJ of parietal epithelial cells of the Bowman's capsule and claudin-2 is present in proximal tubules and thin descending limb of the loop of Henle (30,31). It has been reported that claudin-1 functions as a barrier to ion conductance and to the permeation of 4-40 $\mathrm{kD}$ dextran in vitro, suggesting that it may be responsible for the barrier function of Bowman's capsule $(32,33)$. There are several studies showing that overexpression of the pore-forming claudin-2 in MDCK II or C7 cells preferentially increases the permeability of $\mathrm{Na}^{+}$over $\mathrm{Cl}^{-}(20,34,35)$. We noted similar findings of increased expression of claudin-1 and -2 in NEC kidneys, which may enhance the barrier function of Bowman's capsule and modulate $\mathrm{Na}^{+}$reabsorption to help maintain intravascular volume in NEC cases. 
Claudin-3 is expressed in the thick and thin ascending loops of Henle, distal convoluted tubules and collecting ducts (30). Claudin-3 has been shown to have a sealing function against the passage of small ions of both charged or uncharged solutes, and mainly acts as a general barrier forming protein (36). In contrast, Claudin-7 is mainly expressed in the distal convoluted tubules and collecting ducts, which is the aldosterone sensitive segment of the nephron and plays a vital role in the control of extracellular volume $(19,25,37)$. Deletion of claudin-7 leads to the dehydration and salt wasting phenotype in mice (38). Moderately increased claudin-7 in NEC kidneys suggests that the kidneys may attempt to retain water and modulate the sodium and chloride levels in the body.

Aquaporin-2 (AQP2) is a water channel that plays a vital role in water balance regulation. It is expressed mainly in the collecting ducts and is regulated via the action of vasopressin. Our results showed that AQP2 was significantly increased by both western blot analysis and immunofluorescent staining. This suggests that in NEC pups suffering from decreased intravascular volume, the kidneys try to increase reabsorption of water to maintain intravascular volume. On the other hand, changes in the AQP2 expression could also be due to changes in systemic osmotic pressure induced by formula feeding and/ or changes in intestinal absorption.

In conclusion, our study demonstrates for the first time that NEC leads not only to inflammation in the GI tract, but also renal inflammation, apoptosis and altered expression of several TJ proteins, which may help explain the clinical features such as oliguria, hyponatremia, and fluid imbalance seen in human neonates with NEC.

\section{METHODS}

\section{Generation of Neonatal NEC Mouse Model}

Four-day-old C57BL/6 pups were divided into two groups: the control group was dam-fed and the experimental group was fed with $50 \mu \mathrm{l}$ of $33 \%$ Esbilac formula (Pet-Ag, New Hampshire, IL) every $3 \mathrm{~h}$ for $96 \mathrm{~h}$ using a 26-gauge feeding tube. NEC was induced in the experimental group of pups using a modification of the model described by Jilling et al. (27) where pups fed Esbilac formula were subjected to asphyxia $(100 \% \mathrm{~N} 2$ for $60 \mathrm{~s})$ and cold stress $\left(4^{\circ} \mathrm{C}\right.$ for $\left.10 \mathrm{~min}\right)$ twice a day for 4 $\mathrm{d}(27,39)$. Pups were nursed in an incubator $\left(37^{\circ} \mathrm{C}\right)$ during the 4 - $\mathrm{d}$ period and provided caloric intake of $\sim 800 \mathrm{KJ} / \mathrm{kg}$ per day. In both experimental and control groups, pups were euthanized by $96 \mathrm{~h}$ after the treatments. The gastrointestinal tract and kidneys were carefully removed and visually evaluated for signs of NEC (areas of bowel necrosis, intestinal hemorrhage, perforation). NEC was confirmed by published tissue injury criteria (40). The severity of NEC was graded histologically as follows: grade 1, sloughing of epithelial cells at tip of villi; grade 2, sloughing of epithelial cells to the mid villus level; grade 3 , necrosis of the entire villus; grade 4, transmural necrosis (40). Kidney and intestinal samples were collected for histopathological, cellular, and molecular studies. Our Animal Use Protocol was approved by the Animal Care and Use Committee of East Carolina University.

\section{Antibodies and Reagents}

The rabbit polyclonal anti-claudin-1, -2, -3, -4, -10, and anti-occludin antibodies were purchased from Invitrogen (Carlsbad, CA). The rabbit polyclonal anti-claudin-7 antibody was obtained from Immuno-Biological Laboratories (Hokkaido, Japan). The rabbit anticlaudin-8 antibody was generated in this laboratory (41). The antibodies against ERK1/2, p38, TGF $\beta$, NFкB p65, and Glyceraldehyde 3-phosphate dehydrogenase (GAPDH) were purchased from Cell Signaling Technology (Beverly, MA). The anti-monocyte/macrophage antibody CD11b was from Milenyi Biotech (Auburn, CA). The antiAQP2 antibody was purchased from CALBIOCHEM (Billerica, MA). The Cy3-conjugated anti-mouse IgG and Fluorescein isothiocyanate (FITC)-conjugated goat anti-rabbit IgG were obtained from Jackson ImmunoResearch (West Grove, PA) and EMD Millipore (Billerica, MA), respectively. The horseradish peroxidase (HRP)-conjugated anti-rabbit and anti-mouse secondary antibodies were purchased from Promega (Madison, WI). All other chemicals and reagents were from Sigma (St. Louis, MO) unless indicated otherwise.

\section{Histology Examinations}

Mouse kidneys and intestines from both control and experimental groups were removed from the body after experiments and fixed with $10 \%$ buffered Formalin overnight at $4{ }^{\circ} \mathrm{C}$. Intestines were carefully arranged in a Swiss roll orientation. Subsequently, tissues were rinsed with phosphate-buffered saline and processed through graded ethanol solutions. Paraplast (Fisher, Pittsburgh, PA) embedded tissues were cut on a microtome at 5- $\mu \mathrm{m}$ thickness and sections were placed onto Unifrost Plus glass microscope slides (Azer Scientific, Morgantown, PA). Slides were dried overnight at $43{ }^{\circ} \mathrm{C}$ and stored at room temperature until stained. Hematoxylin \& Eosin (H\&E) and Periodic acid Schiff (PAS) staining was performed according to Armed Forces Institute of Pathology Laboratory methods in Histotechnology (42).

\section{Western Blot Analysis}

Kidney tissues from control and NEC pups were minced on ice and then sonicated in ice cold RIPA buffer (1\% Triton X-100, $0.5 \%$ sodium deoxycholate, $0.2 \% \mathrm{SDS}, 150 \mathrm{mmol} / \mathrm{l} \mathrm{NaCl}, 10 \mathrm{mmol} / \mathrm{l} \mathrm{N}-2$ hydroxyethylpiperazine- $\mathrm{N}$-2-ethane sulfonic acid, pH7.3, $2 \mathrm{mmol} / \mathrm{l}$ Ethylenediaminetetraacetic acid, $10 \mu \mathrm{g} / \mathrm{ml}$ each of chymostatin, leupeptin, and pepstatin A) to obtain tissue lysates. The total protein concentration of each sample was measured using the BCA protein assay kit (Pierce laboratories, Rockford, IL) and adjusted to equal concentration $(2 \mathrm{mg} / \mathrm{ml})$. Proteins were separated by SDS-PAGE and transferred to nitrocellulose membranes. Membranes were then blocked in $5 \%$ nonfat dry milk in phosphate-buffered saline plus $0.1 \%$ Tween 20 and incubated with primary antibodies at $4{ }^{\circ} \mathrm{C}$ overnight followed by incubation with appropriate secondary antibodies for $1 \mathrm{~h}$ at room temperature. After blotting, the signals were detected by enhanced chemiluminescence (Amersham, Arlington Heights, IL) on Blue Devil autoradiography film (Genesee Scientific, San Diego, CA).

\section{Immunofluorescence Microscopy}

Frozen kidney sections were fixed in $100 \%$ acetone for $5 \mathrm{~min}$ at -20 ${ }^{\circ} \mathrm{C}$, and then washed with phosphate-buffered saline before blocking in $5 \% \mathrm{BSA}$ for $60 \mathrm{~min}$ at room temperature. The tissues were incubated with primary antibodies followed by incubation with appropriate secondary antibody for $60 \mathrm{~min}$ at room temperature. Coverslips were mounted with ProLong Antifade Kit (Molecular Probes, Eugene, OR). Samples were analyzed and photographed using a Zeiss Axio Imager M2 microscope (Carl Zeiss, Thornwood, NY) equipped with AxioVision imaging software.

\section{ACKNOWLEDGMENTS}

We thank Beverly G. Jeansonne and Joani Zary Oswald for their technical assistance.

\section{STATEMENT OF FINANCIAL SUPPORT}

This study was supported by National Institutes of Health (Bethesda, MD) grant HL085752 to Y.-H.C.

Disclosure: The authors have nothing to disclose.

\section{REFERENCES}

1. Jain L. Necrotizing enterocolitis prevention: art or science? Clin Perinatol 2013;40:xiii-Xv.

2. Petrosyan M, Guner YS, Williams M, Grishin A, Ford HR. Current concepts regarding the pathogenesis of necrotizing enterocolitis. Pediatr Surg Int 2009;25:309-18.

3. Neu J, Walker WA. Necrotizing enterocolitis. N Engl J Med 2011;364: $255-64$.

4. Stoll BJ, Hansen NI, Bell EF, et al.; Eunice Kennedy Shriver National Institute of Child Health and Human Development Neonatal Research Net- 


\section{Articles | Garget al.}

work. Neonatal outcomes of extremely preterm infants from the NICHD Neonatal Research Network. Pediatrics 2010;126:443-56.

5. Denning PW, Maheshwari A. Necrotizing enterocolitis: hope on the horizon. Clin Perinatol 2013;40:xvii-xix.

6. Piena-Spoel M, Albers MJ, ten Kate J, Tibboel D. Intestinal permeability in newborns with necrotizing enterocolitis and controls: Does the sugar absorption test provide guidelines for the time to (re-)introduce enteral nutrition? J Pediatr Surg 2001;36:587-92.

7. Clark JA, Doelle SM, Halpern MD, et al. Intestinal barrier failure during experimental necrotizing enterocolitis: protective effect of EGF treatment. Am J Physiol Gastrointest Liver Physiol 2006;291:G938-49.

8. Feng J, El-Assal ON, Besner GE. Heparin-binding epidermal growth factor-like growth factor decreases the incidence of necrotizing enterocolitis in neonatal rats. J Pediatr Surg 2006;41:144-9; discussion 144-9.

9. Arrieta MC, Bistritz L, Meddings JB. Alterations in intestinal permeability. Gut 2006;55:1512-20.

10. Bergmann KR, Liu SX, Tian R, et al. Bifidobacteria stabilize claudins at tight junctions and prevent intestinal barrier dysfunction in mouse necrotizing enterocolitis. Am J Pathol 2013;182:1595-606.

11. Viswanathan VK, Weflen A, Koutsouris A, Roxas JL, Hecht G. Enteropathogenic E. coli-induced barrier function alteration is not a consequence of host cell apoptosis. Am J Physiol Gastrointest Liver Physiol 2008;294:G1165-70.

12. Eadon MT, Hack BK, Xu C, Ko B, Toback FG, Cunningham PN. Endotoxemia alters tight junction gene and protein expression in the kidney. Am J Physiol Renal Physiol 2012;303:F821-30.

13. Angelow S, Ahlstrom R, Yu AS. Biology of claudins. Am J Physiol Renal Physiol 2008;295:F867-76.

14. González-Mariscal L, Betanzos A, Nava P, Jaramillo BE. Tight junction proteins. Prog Biophys Mol Biol 2003;81:1-44.

15. Schneeberger EE, Lynch RD. The tight junction: a multifunctional complex. Am J Physiol Cell Physiol 2004;286:C1213-28.

16. Van Itallie CM, Anderson JM. Claudins and epithelial paracellular transport. Annu Rev Physiol 2006;68:403-29.

17. Heiskala M, Peterson PA, Yang Y. The roles of claudin superfamily proteins in paracellular transport. Traffic 2001;2:93-8.

18. Alexandre MD, Jeansonne BG, Renegar RH, Tatum R, Chen YH. The first extracellular domain of claudin-7 affects paracellular $\mathrm{Cl}$ - permeability. Biochem Biophys Res Commun 2007;357:87-91.

19. Alexandre MD, Lu Q, Chen YH. Overexpression of claudin-7 decreases the paracellular $\mathrm{Cl}$ - conductance and increases the paracellular $\mathrm{Na}+$ conductance in LLC-PK1 cells. J Cell Sci 2005;118(Pt 12):2683-93.

20. Amasheh S, Meiri N, Gitter AH, et al. Claudin-2 expression induces cation-selective channels in tight junctions of epithelial cells. J Cell Sci 2002;115(Pt 24):4969-76.

21. Colegio OR, Van Itallie CM, McCrea HJ, Rahner C, Anderson JM. Claudins create charge-selective channels in the paracellular pathway between epithelial cells. Am J Physiol Cell Physiol 2002;283:C142-7.

22. Hou J, Gomes AS, Paul DL, Goodenough DA. Study of claudin function by RNA interference. J Biol Chem 2006;281:36117-23.

23. Szaszi K, Amoozadeh Y. New insights into functions, regulation, and pathological roles of tight junctions in kidney tubular epithelium. Int Rev Cell Mol Biol 2014;308:205-71.
24. Muto S, Furuse M, Kusano E. Claudins and renal salt transport. Clin Exp Nephrol 2012;16:61-7.

25. Hou J. Regulation of paracellular transport in the distal nephron. Curr Opin Nephrol Hypertens 2012;21:547-51.

26. Li J, Ananthapanyasut W, Yu AS. Claudins in renal physiology and disease. Pediatr Nephrol 2011;26:2133-42.

27. Jilling T, Simon D, Lu J, et al. The roles of bacteria and TLR4 in rat and murine models of necrotizing enterocolitis. J Immunol 2006;177: 3273-82.

28. Trujillo J, Molina-Jijón E, Medina-Campos ON, et al. Renal tight junction proteins are decreased in cisplatin-induced nephrotoxicity in rats. Toxicol Mech Methods 2014;24:520-8.

29. Molina-Jijón E, Rodríguez-Muñoz R, Namorado Mdel C, PedrazaChaverri J, Reyes JL. Oxidative stress induces claudin-2 nitration in experimental type 1 diabetic nephropathy. Free Radic Biol Med 2014;72: $162-75$.

30. Kiuchi-Saishin Y, Gotoh S, Furuse M, Takasuga A, Tano Y, Tsukita S. Differential expression patterns of claudins, tight junction membrane proteins, in mouse nephron segments. J Am Soc Nephrol 2002;13:875-86.

31. Ohse T, Pippin JW, Vaughan MR, Brinkkoetter PT, Krofft RD, Shankland SJ. Establishment of conditionally immortalized mouse glomerular parietal epithelial cells in culture. J Am Soc Nephrol 2008;19: 1879-90.

32. Inai T, Kobayashi J, Shibata Y. Claudin-1 contributes to the epithelial barrier function in MDCK cells. Eur J Cell Biol 1999;78:849-55.

33. McCarthy KM, Francis SA, McCormack JM, et al. Inducible expression of claudin-1-myc but not occludin-VSV-G results in aberrant tight junction strand formation in MDCK cells. J Cell Sci 2000;113 Pt 19:3387-98.

34. Furuse M, Furuse K, Sasaki H, Tsukita S. Conversion of zonulae occludentes from tight to leaky strand type by introducing claudin-2 into MadinDarby canine kidney I cells. J Cell Biol 2001;153:263-72.

35. Yu AS, Cheng MH, Angelow S, et al. Molecular basis for cation selectivity in claudin-2-based paracellular pores: identification of an electrostatic interaction site. J Gen Physiol 2009;133:111-27.

36. Milatz S, Krug SM, Rosenthal R, et al. Claudin-3 acts as a sealing component of the tight junction for ions of either charge and uncharged solutes. Biochim Biophys Acta 2010;1798:2048-57.

37. Li WY, Huey CL, Yu AS. Expression of claudin-7 and -8 along the mouse nephron. Am J Physiol Renal Physiol 2004;286:F1063-71.

38. Tatum R, Zhang Y, Salleng K, et al. Renal salt wasting and chronic dehydration in claudin-7-deficient mice. Am J Physiol Renal Physiol 2010;298: F24-34.

39. Radulescu A, Zhang HY, Yu X, et al. Heparin-binding epidermal growth factor-like growth factor overexpression in transgenic mice increases resistance to necrotizing enterocolitis. J Pediatr Surg 2010;45:1933-9.

40. Caplan MS, Hedlund E, Adler L, Hsueh W. Role of asphyxia and feeding in a neonatal rat model of necrotizing enterocolitis. Pediatr Pathol 1994;14:1017-28.

41. Jeansonne B, Lu Q, Goodenough DA, Chen YH. Claudin-8 interacts with multi-PDZ domain protein 1 (MUPP1) and reduces paracellular conductance in epithelial cells. Cell Mol Biol (Noisy-le-grand) 2003;49:13-21.

42. Prophet E, editor. Laboratory methods in Histotechnology. Washington, DC: American Registry of Pathology, 1992. 